\title{
Prevalence and Microbial Flora of Chicken Slaughtering and Processing Procedure
}

\author{
Kuk-Hwan Seol, Gi-Sung Han, Hyoun Wook Kim, Oun-Ki Chang, Mi-Hwa Oh, \\ Beom-Young Park, and Jun-Sang Ham* \\ National Institute of Animal Science, Rural Development Administration, Suwon 441-706, Korea
}

\begin{abstract}
This study has been performed to measure the prevalence and microbial flora on chicken slaughtering as well as the processing process from the months of October to November. Whole-chicken rinsing technique was used in order to analyze the incidence of microorganisms on chicken carcass at the stage before chilling (after evisceration), after chilling and after cutting. The swab technique was used on processing the processed samples, such as working plates and cutting knives. Brine and cooling water from four cooling tubs were taken from each processing processes and were used as samples. Furthermore, the matrix-assisted laser desorption/ionization mass spectrometry (MALDI MS) for whole cell fingerprinting in combination with a dedicated bioinformatic software tool was used to identify the isolated microorganisms. Of the tested samples and processes, brine $(4.50 \pm 0.64 \mathrm{Log}$ CFU/mL) and chicken carcass before chilling $(4.15 \pm 0.46 \mathrm{Log}$ CFU/mL) showed the highest population of microorganisms; the predominant microbial flora of them were Moellerella wisconsensis (54.84\%), a member of the Enterobacteriaceae family, and Escherichia coli (60.36\%), respectively. However, the predominant microbial flora of cut carcass was changed to Staphylococcus aureus $(27.32 \%)$, which is a kind of pathogenic microorganism that can cause a food-borne illness. Therefore, the slaughtering and processing procedure of chicken are needed to be controlled more hygienically.
\end{abstract}

Key words: chicken slaughtering, aerobic bacteria, microbial flora, MALDI-TOF MS

\section{Introduction}

The microbiological status of processed poultry carcasses has been reported to be dependent on several key factors, such as the level of contamination from live birds, numbers and genera of pathogenic or indicator organisms introduced at pre-harvest phases, and the extent of occurrence of contamination and cross-contamination during processing (Abu-Ruwaida et al., 1994). The control of contamination during slaughter and processing has been identified as critical in relation to the prevalence of pathogenic microorganisms on end products (Mead et al., 1993). The mechanisms by which contamination occurs during processing, other than immersion in scald and chill waters, have been described by Schuler and Badenhop (1972) and Carson et al. (1987), who demonstrated carcass-carcass and equipment-carcass contacts

*Corresponding author: Jun-Sang Ham, National Institute of Animal Science, Rural Development Administration, Suwon 441706, Korea. Tel: 82-31-290-1692, Fax: 82-31-290-1697, E-mail: hamjs@korea.kr to be significant routes in the spread of pathogenic organisms. Upton (1996) noted that the extent to which contamination occurred on carcasses and the composition of the resulting flora reflected the hygiene standards at processing level, together with the initial microbiological status of the animal. Smoot and Pierson (1997) regarded the application of microbiological criteria using indicator organisms as useful for foods such as poultry which were repeatedly implicated in food borne disease outbreaks, as the presence of certain organisms indicated the possibility of occurrence of microbiological hazards. The potential microbiological hazards in meat products cannot be assessed nor demonstrated reliably with the use of acceptable levels based on total counts or indicator organisms (Brown and Baird-Parker, 1982). However, the analysis of foods of animal origin for the presence or levels of generic Escherichia coli has been widely accepted as an indicator of faecal contamination, while failure to detect this organism cannot guarantee the absence of other enteric pathogens (Brown and Baird-Parker, 1982; Silliker and Gabis, 1976). Total aerobic counts have been recommended as a useful tool in the microbiological 
assessment of food safety, as high mesophilic counts (25$37^{\circ} \mathrm{C}$ ) may indicate unsatisfactory sanitation or the use of heavily contaminated raw materials (Thatcher and Clark, 1968). Lillard et al. (1984) and Al-Mohizea et al. (1994) also acknowledged the benefit of aerobic plate counts as a criterion in assessing the hygiene of poultry processing plants.

Developments in a number of mass spectrometry (MS) techniques in recent years have provided opportunities to evaluate MS for the identification of microorganisms (Fenselau, 1994). Matrix-assisted laser desorption ionization time-of-flight mass spectrometry (MALDI-TOF-MS) has a number of advantages over other methods of mass spectrometry when analyzing biological material. The great advantage of MALDI-TOF-MS when working with microorganisms is that intact cells can be taken directly from a colony and analyzed within minutes. Intact cell MALDI-TOF-MS, also known as ICM-MS, produces a mass spectral fingerprint of moieties desorbed from the cell surface (Claydon et al., 1996; Holland et al., 1996; Welham et al., 1998), and therefore, has the added advantage that the location of the source of ions is known. Identification by ICM-MS may relate to conventional phenotypic identification methods of identification from Gram staining to serotyping or phage typing (EdwardsJones et al., 2000), as these are dependant upon cell surface components or morphology. In addition, sufficient structural information can be obtained by ICM-MS, particularly in conjunction with post source decay, to determine the identification of the moieties produced by this method (Leenders et al., 1999).

This study was performed to analyze the prevalence and microbial flora of chicken slaughtering and processing procedure. In addition, the study showed the possibility of analyzing the microbial flora using MALDI-TOFMS in combination with a dedicated bioinformatic software tool.

\section{Material and Methods}

\section{Collection of samples}

A large scale (more than 100,000 heads per day) poultry slaughter house was visited 3 times to take samples of chicken carcasses and processing processes from October to November. Chicken carcasses of before washing (after evisceration), after chilling and cut carcasses (15 carcasses for each step) were taken to analyze the incidence of microorganisms. Each chicken carcass sample was put into a sterilized poultry rinsing bag, then sealed and kept in ice-box for transportation to laboratory at below $4^{\circ} \mathrm{C}$. Samples of processing, such as cooling water, cutting knife, brine and conveyor belt, were also taken into sterilized tube and swab kit (Environmental swab, $3 \mathrm{M}^{\mathrm{TM}}$, China) and transported to laboratory at below $4^{\circ} \mathrm{C}$.

\section{Microbiological Analysis}

Each carcass samples were added $400 \mathrm{~mL}$ of $0.1 \%$ peptone water (Difco Laboratories, USA) and shook for $1 \mathrm{~min}$. Subsequently, $40 \mathrm{~mL}$ of peptone water was taken and serial 10 -fold dilution was prepared by transferring $1 \mathrm{~mL}$ of this solution to $9 \mathrm{~mL}$ tubes of $0.1 \%$ peptone water. Processing samples were took $1 \mathrm{~mL}$ serially diluted in the same way and used for the microbiological analysis.

Once samples had been prepared, the levels of total aerobic bacteria and E. coli were established. For total aerobic and $E$. coli counts, $1 \mathrm{~mL}$ volumes of relevant dilutions were placed in duplicate plates and approximately $20 \mathrm{~mL}$ of nutrient agar (Difco Laboratories, USA) were added and allowed to solidity. Plates were incubated at $37^{\circ} \mathrm{C}$ for $24 \mathrm{~h}$ and the plates had 30 to 300 colonies were selected for counting and screening.

\section{Matrix and standard preparation}

A HCCA matrix ( $\alpha$-cyano-4-hydroxy-cinnamic acid) (Part NO., 255344, Bruker Daltonik GmbH, Germany) was used as a saturated solution in a solvent mixture. Before each MALDI run E. coli DH5 $\alpha$ was measured to serve as the positive control and calibration standard. Known ribosomal proteins of $E$. coli were employed for the task of calibration (RL36, RS22, RL34, RL33meth, RL32, RL29, and RS19).

\section{Mass spectrometry}

The MALDI measurements were performed on a Bruker Microflex TOF mass spectrometer (Bruker Daltonik GmbH, Germany) equipped with a nitrogen laser. Microorganisms were used directly from fresh colonies as follow; $1 \mu \mathrm{g}$ of microorganisms were put on the plate and dried in room temperature, then $2 \mu \mathrm{L}$ of HCCA matrix was added on the microorganism spot and dried for analysis. The instruments are fitted with a pulsed nitrogen laser $(337 \mathrm{~nm})$ with a 3-ns pulse duration. Spectra were recorded in the linear positive mode within a mass range of 2,000-20,000 Da. The initial "matrix blast" considerable increased the quality of the spectra acquired. The acquisition of spectra was performed both manually and automatically just above the "desorption level" of laser energy to maximize 
the resolution of the peak pattern. A minimum resolution of 600 had to be reached within the range of 4,00012,000 Da. The acquisition of spectra was performed both manually and automatically using the Bruker flexControl in the autoexecute mode. The spectra were collected as a sum of 10 times 50 laser shots ranged across a target spot. Twenty independent spectra were collected for each isolated microorganism.

\section{Data processing using Bruker BioTyper ${ }^{\mathrm{TM}}$ and identification of isolates}

The spectra were analyzed using Bruker BioTyper ${ }^{\mathrm{TM}}$ 2.0 software, whereby raw spectra were directly handled by the program. The spectra were subsequently smoothed and baseline correctred. In addition to the afore-mentioned processes, the peaks were selected. Using peak lists of approximate 20 spectra as a basis, a reference peak list (the main spectrum) of each microorganism was calculated. The threshold for peak acceptance was set at $\mathrm{S} / \mathrm{N}$ (signal to noise) of two. The spectra were aligned with each other before the reference spectra were calculated. After alignment, peaks with an $\mathrm{m} / \mathrm{z}$ (mass-to-charge ratio) difference of less than $250 \mathrm{ppm}$ were considered to be identical.

\section{Statistical analysis}

All bacterial counts obtained from chicken slaughtering and processing process were transformed to $\log _{10}$ values for subsequent data analysis. Statistical analysis was performed with the SAS program for Windows V9.2 (SAS Institute, USA). General linear model (GLM) with Duncan's multiple range test was carried out to analyze the significant differences among the processes $(p<0.05)$.

\section{Results and Discussion}

\section{The microbial counts of chicken slaughtering and processing}

The mean aerobic bacterial counts in chicken slaughtering and processing procedure include chicken carcass and cuts are shown in Fig. 1. The brine showed the highest bacterial count $(4.50 \pm 0.64 \mathrm{Log} \mathrm{CFU} / \mathrm{mL})$ followed by chicken carcass before chilling $(4.15 \pm 0.46 \mathrm{Log}$ CFU $/ \mathrm{mL})$, $2^{\text {nd }}$ cutting knife $(3.83 \pm 0.67 \mathrm{Log} C \mathrm{FU} / \mathrm{mL})$ and cut carcass (3.77 $\pm 0.49 \mathrm{Log} \mathrm{CFU} / \mathrm{mL})$ while working plate declared the lowest counts $\left(0.30 \pm 0.00 \mathrm{Log} \mathrm{CFU} / \mathrm{cm}^{2}\right)$ population. Among brine, chicken carcass before chilling, $2^{\text {nd }}$ cutting knife and cut carcass, no significant differences in the bacterial counts were detected $(p>0.05)$. The bacterial counts of chicken carcass was significantly decreased after four stage of water immersion chilling from $4.15 \pm$ $0.46 \mathrm{Log} \mathrm{CFU} / \mathrm{mL}$ (chicken carcass before chilling) to $2.50 \pm 0.36 \mathrm{Log} \mathrm{CFU} / \mathrm{mL}$ (chicken carcass after chilling, $p<0.05$ ), however after brine injection and cutting, the bacterial counts were increased to $3.77 \pm 0.49 \mathrm{Log} \mathrm{CFU} /$ $\mathrm{mL}$ (cut carcass, $p<0.05$ ).

\section{Microbial flora of chicken slaughtering and pro- cessing procedure}

The microbial flora of chicken slaughtering and processing processes analyzed by MALDI-TOF MS system were shown in Fig. 3. There exist various kinds of microorganisms, 13 and 10 kinds from chicken carcasses before and after chilling, respectively, 13 kinds from chicken cuts, 6 kinds from brine, 7 kinds from chilling water, and 15 kinds from cutting knives. The predominant microbial flora of chicken carcasses of before $(60.36 \%)$ and after

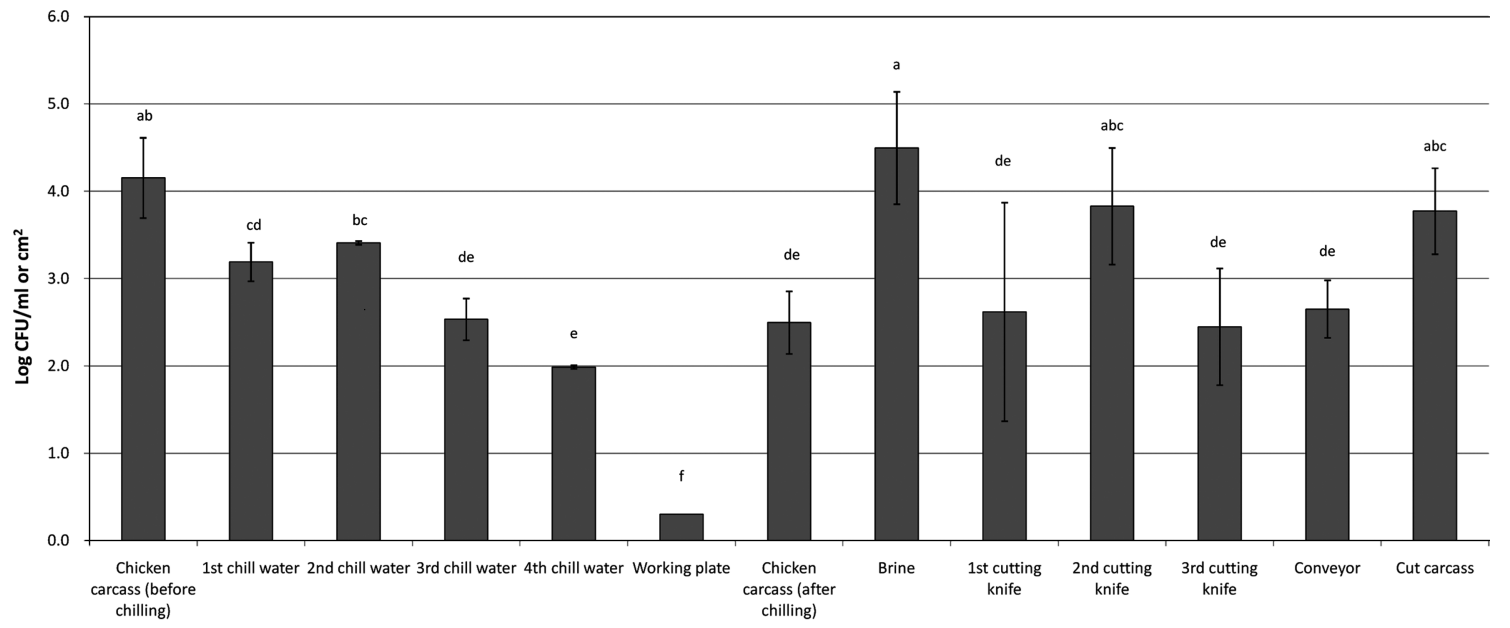

Fig. 1. The aerobic bacterial counts of chicken slaughtering and processing process. Values represent means $\pm \mathrm{SE}$ of samples. Bars with different superscript are significantly different $(p<0.05)$. 
a)
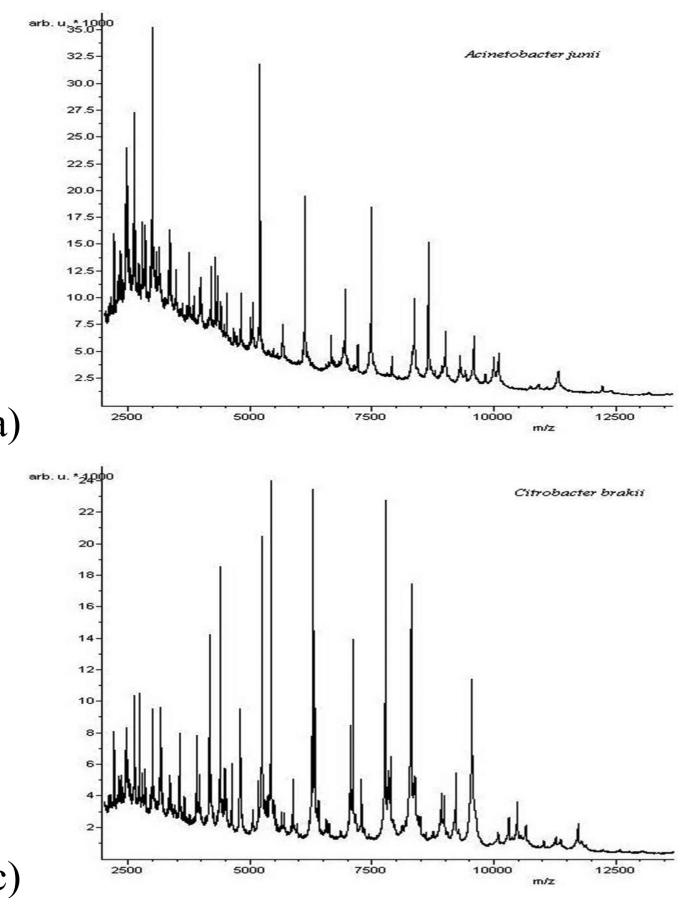

c)

e)

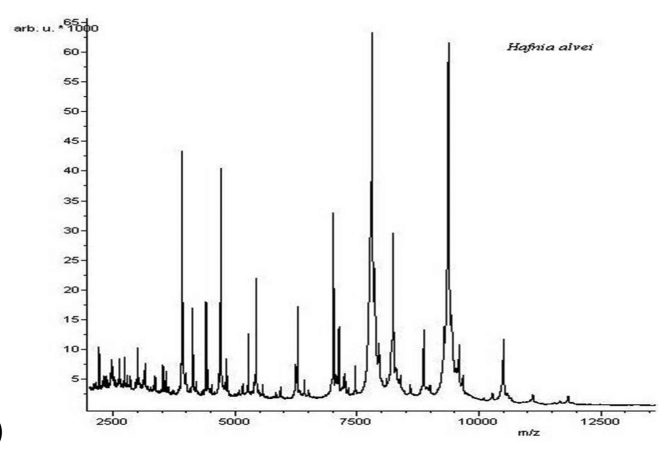

g)

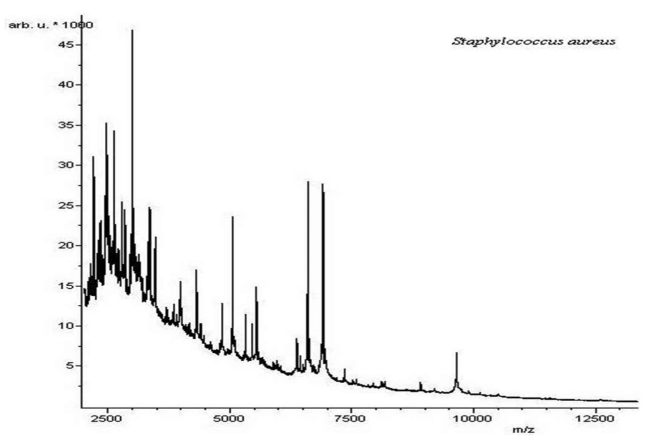

b)

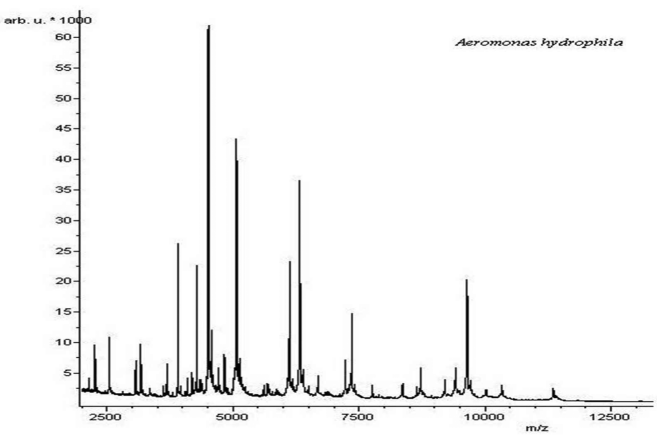

d)

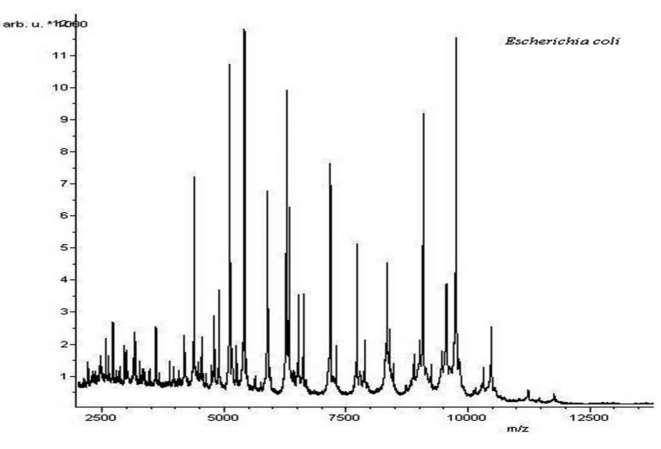

f)

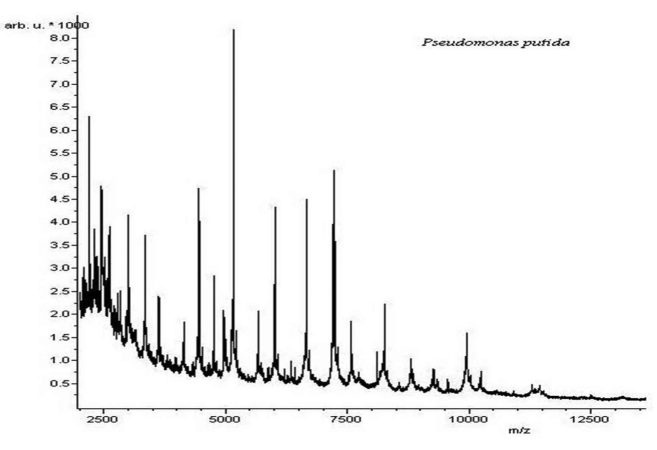

h)

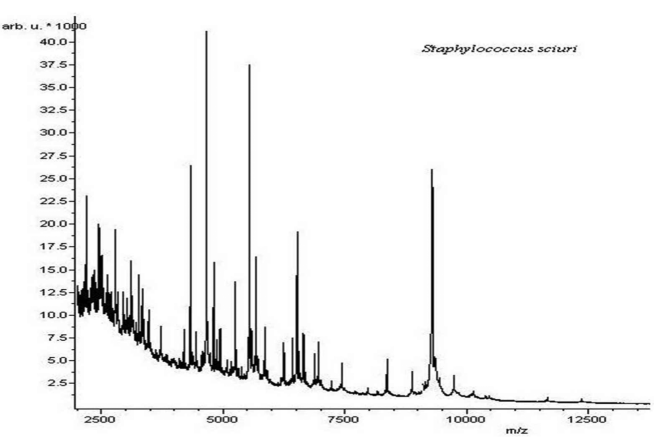

Fig. 2. Typical matrix-assisted laser desorption/ionization (MALDI) fingerprint spectra of several isolated microorganisms. a, Acinetobacter junii; b, Aeromonas hydrophila; c, Citrobacter brakii; d, Escherichia coli; e, Hafnia alvei; f, Pseudomonas putida; g, Stephylococcus aureus; h, Staphylococcus sciuri

chilling (34.27\%) was Escherichia coli (Fig. 3a and b). However, after cut the chicken carcass, the predominant microbial flora was changed to Staphylococcus aureus $(27.32 \%$, Fig. 3c). In the brine, which showed the highest population of aerobic microorganism, the predominant microbial flora was Moellerella wisconsensis (54.84\%), a member of the Enterobacteriaceae family. E. coli is known as a major indicator microorganism of fecal contamination and $S$. aureus is a kind of pathogenic microorganism that can cause a food-borne illness. In this study, it was not analyzed the amount of $E$. coli and $S$. aureus. However, they are widely spread in the chicken slaughtering and processing processes and the ratios of them were relatively higher than other microorganisms in the microbial 

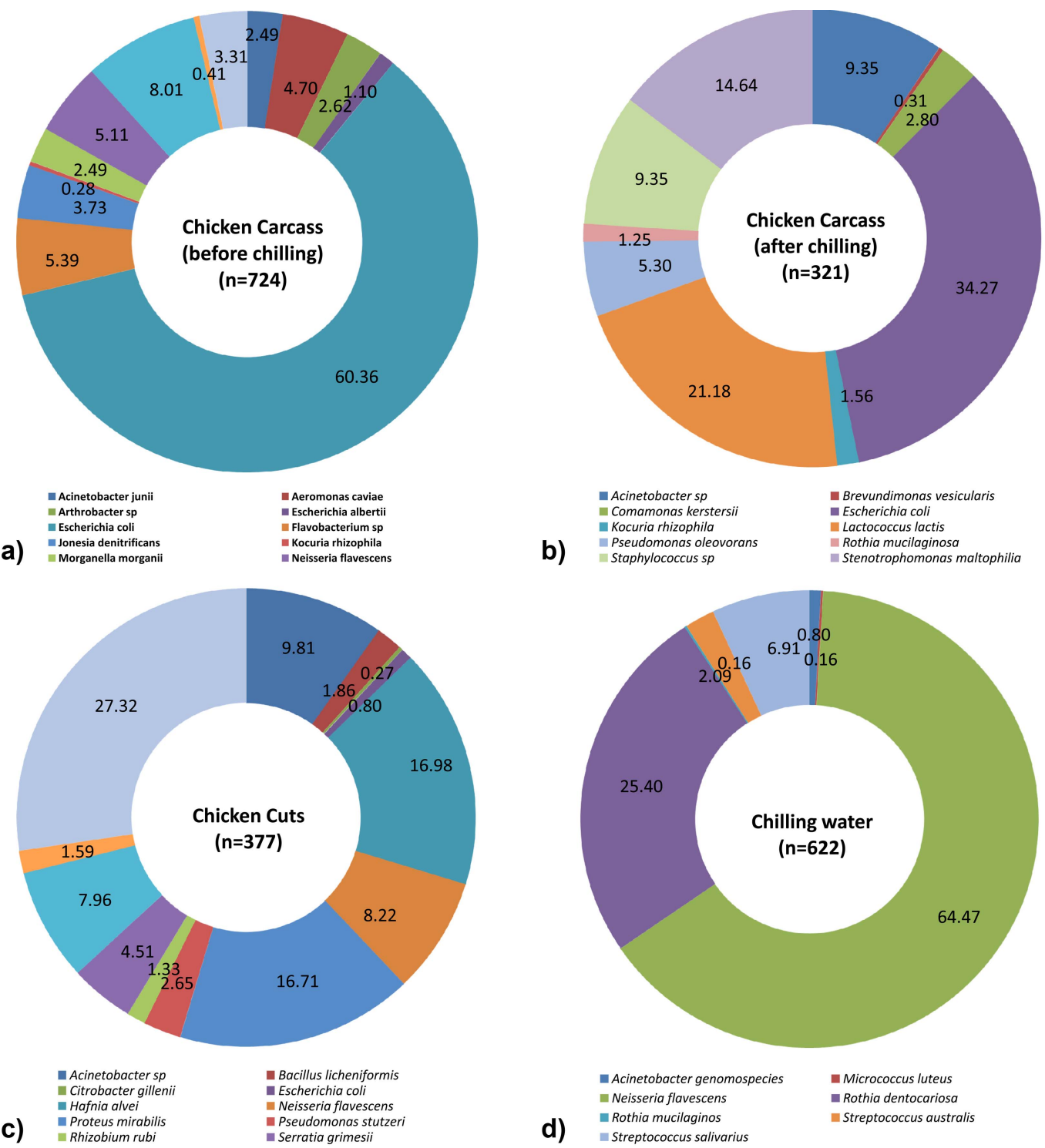

$\begin{array}{ll}\text { b) } & \text { Pseudomonas oleovorans } \\ \text { Staphylococcus sp } & \text { Rothia mucilaginosa } \\ \text { Stenotrophomonas maltophilia }\end{array}$
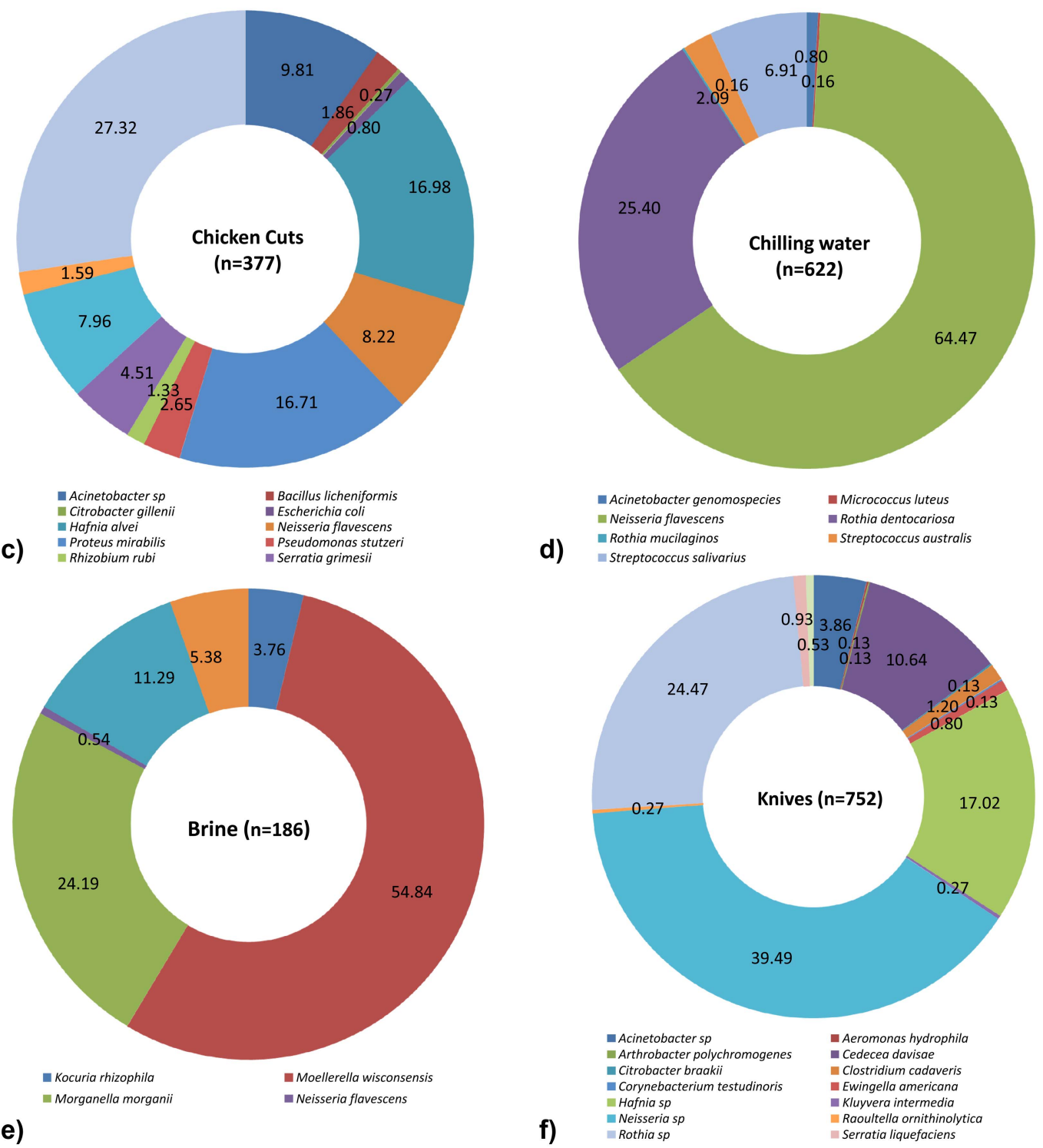

d) Rothia mucilaginos $\quad$ Streptococcus australis

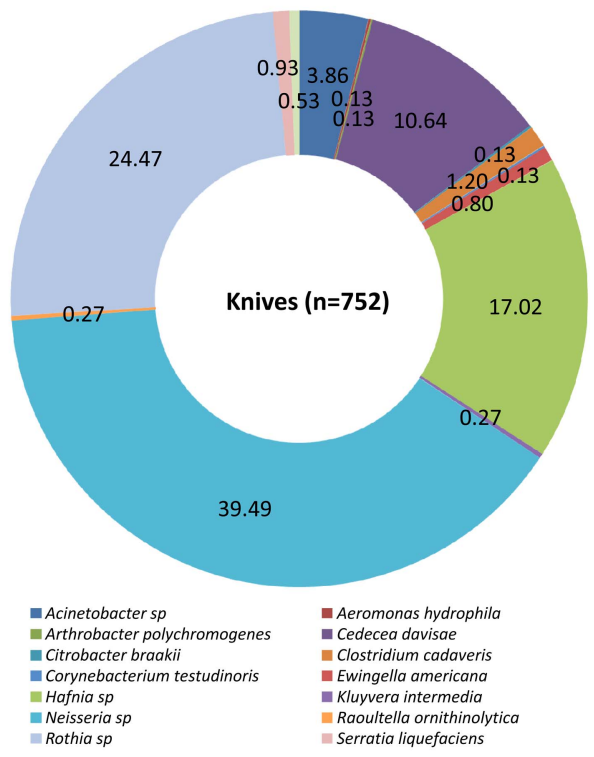

Fig. 3. Microbial flora of chicken slaughtering and processing process. a, chicken carcass (before chilling); b, chicken carcass (after chilling); c, chicken cuts; d, brine; e, chilling water; $f$, knives 
flora. Therefore, the slaughtering and processing procedure of chicken are needed to be controlled more hygienically.

\section{Conclusion}

This study has provided the possibility of MALDI-TOF MS for a rapid, comparatively cheap and reliable identification of bacterial isolates from chicken slaughtering and processing process. Additional study with MALDI-TOF MS, coupled with corresponding phylogenetic analysis, may allow better insight in the spread of microorganisms within the chicken slaughtering process.

\section{Acknowledgement}

This work was sponsored by project PJ007335 of Rural Development Administration of Korea.

\section{References}

1. Abu-Ruwaida, A. A., Sawaya, W. N., Dashti, B. H., Murad, M. and Al-Othman, H. A. (1994) Microbiological quality of broilers during processing in a modern commercial slaughterhouse in Kuwait. J. Food Prot. 57, 887-892.

2. Al-Mohizea, I. S., Mashhadi, A. S., Fawwal, A. and Al-Shalhat, A. (1994) Microbiological and shelf life assessment of chilled eviscerated whole chicken broilers in Saudi Arabia. Br. Poult. Sci. 35, 519-526.

3. Brown, M. H. and Baird-Parker, A. C. (1982) The microbiological examination of meat. In: Brown, M. H. (Ed.), Meat Microbiology. Applied Science Publishers, London, pp. 423520.

4. Carson, M. O., Lillard, H. S. and Hamdy, M. K. (1987) Transfer of firmly attached ${ }^{32} \mathrm{P}$-Salmonella typhimurium from raw poultry skin to other surfaces. J. Food Prot. 50, 327-329.

5. Claydon, M. A., Davey, S. N., Edward-Jones, V. and Gordon, D. B. (1996) The rapid identification of intact microorganisms using mass spectrometry. Nat. Biotechnol. 14, 15841586.

6. Edwards-Jones, V., Claydon, M. A., Evason, D. J., Walker, J., Fox, A. J. and Gordon, D. B. (2000) Rapid discrimination between methicillin-sensitive and methicillin-resistant Staphylococcus aureus by intact cell mass spectrometry. J. Med. Microbiol. 49, 1-6.
7. Fenselau, C. (1994) Mass spectrometry for the characterization of microorganisms. ACS Symposium Series, vol. 541. ACS, Washington.

8. Holland, R. D., Wikes, J. G., Rafii, F., Sutherland, J. B., Persons, C. C., Voorhees, K. H. and Lay, J. O. (1996) Rapid identification of intact whole bacteria based on spectral patterns using matrix-assisted laser desorption with time-offlight mass spectrometry. Rapid Commun. Mass Spectrom. 10, 1227-1232.

9. Leenders, K., Stein, T. H., Kablitz, B., Franke, P. and Vater, J. (1999) Rapid typing of Bacillus subtilus strains by their secondary metabolites using matrix-assisted laser desorption/ionization mass spectrometry of intact cells. Rapid Commun. Mass Spectrom. 13, 943-949.

10. Lillard, H. S., Cox, N. A., Bailey, J. S. and Thomson, J. E. (1984) Comparison of brands of media for isolating bacteria from poultry, beef and shrimp. J. Food Prot. 47, 394-397.

11. Mead, G. C., Hudson, W. R. and Hinton, M. H. (1993) Microbiological survey of five poultry processing plants in the UK. Br. Poult. Sci. 34, 497-503.

12. Schuler, G. A. and Badenhop, A. F. (1972) Microbiology survey of equipment in selected poultry processing plants. Poult. Sci. 51, 830-835.

13. Silliker, J. H. and Gabis, D. A. (1976) ICMSF method studies. VII. Indicator tests as substitutes for direct testing of dried foods and feeds for Salmonella. Can. J. Microbiol. 22, 971-974.

14. Smoot, M. L. and Pierson, M. D. (1997) Indicator microorganisms and microbiological criteria. In: Doyle, M. P., Beuchat, L. R., Montville, T. J. (Eds.), Food Microbiology Fundamentals and Frontiers. ASM Press, Washington DC, pp. 66-80.

15. Thatcher, F. S. and Clark, D. S. (1968) Indicator organisms. In: Thatcher, F. S., Clark, D. S. (Eds.), Microorganisms in Foods - Their Significance and Methods of Enumeration. University of Toronto Press, pp. 23-37.

16. Upton, M. (1996) Relationships between pathogenic growth and the general microbiota on raw and processed meat and poultry. In: Sheridan, J. J., Buchanan, R. L., Montville, T. J. (Eds.), HACCP: An Integrated Approach to Assuring the Microbiological Safety of Meat and Poultry. Food and Nutrition Press, Connecticut, pp. 139-150.

17. Welham, K. J., Domin, M. A., Scannell, D. E., Cohen, E., and Ashton, D. S. (1998) The characterization of microorganisms by matrix-assisted laser desorption ionization time-offlight mass spectrometry. Rapid Commun. Mass Spectrum. 12, 176-180.

$\overline{\text { (Received 2012.9.15/Revised 2012.11.9/Accepted 2012.11.21) }}$ 